\section{Desigualdades regionais na prevalência de diagnóstico de asma em crianças: uma análise da Pesquisa Nacional por Amostra de Domicílios, 2003}

\author{
Regional inequalities in the prevalence of asthma \\ diagnosis in children: an analysis of the Brazilian \\ National Household Sample Survey, 2003
}

${ }^{1}$ Centro de Ciências da Saúde, Universidade Federal de Santa Catarina, Florianópolis, Brasil.

Correspondência K. G. A. Peres

Departamento de Saúde Pública, Centro de Ciências da Saúde, Universidade Federal de Santa Catarina. Campus Universitário Trindade, Florianópolis, SC 88010-970, Brasil.

karengp@ccs.ufsc.br
Abstract

The aim of this study was to estimate the prevalence of asthma diagnosis in Brazilian children and to analyze socio-demographic inequalities between the South and Northeast regions of the country. Data for children under 10 years of age were analyzed from the 2003 National Household Sample Survey, or PNAD $(n=69,796)$. Socioeconomic characteristics, the child's gender and age, and the parent or guardian's skin color were the independent variables investigated through un3.5) and 4.4\% (95\% CI:4.0-4.8) in the South and Northeast, respectively. After adjustment, children from 3 to 7 years of age and those from the poorest families were more likely to have an asth ma diagnosis in both regions. Black skin colo crowding and substandard housing were associted with asthma in the South. In the Northeast, children of parents with low schooling were less likely to present asthma. The inequalities were more evident in the South, suggesting variation in asthma determinants across the country.

Asthma; Health Inequalities; Child
Fernando César Wehrmeister 1 Karen Glazer de Anselmo Peres 1

\section{Introdução}

Segundo dados Mundial da Saúde (OMS), cerca de 13 milhões de crianças menores de cinco anos morrem anualmente no mundo por doenças do aparelho respiratório e 95\% destes óbitos ocorrem nos países em desenvolvimento ${ }^{1}$. Dentre as doenças respiratórias, destaca-se a asma.

Asma é uma doença caracterizada por limitação do fluxo nas vias aéreas, sendo considerada dentre as doencas crônicas uma das mais prevalentes na infancia, com importante incremento lentes na infancia, com importante incremento na sua prevalência 2 , principalmente a partir da década de 70 3,4,5. Esse crescimento pode ser explicado pela combinação de fatores ambientais e de estilo de vida 2,6,7. Adicionalmente, a asma pode influenciar negativamente a qualidade de vid das crianças como, por exemplo, no absenteísmo escolar, e afetar a sociedade como um todo devido aos altos custos para o sistema de saúde 4 .

Em uma revisão de literatura a respeito da epidemiologia da asma em crianças, realizada nas bases de dados PubMed, SciELO e LILACS no período de julho de 1998 a julho de 2008, foram encontrados 119 artigos utilizando-se os descritores asthma, bronchitis, prevalence, epidemiology, income, self-rated, gender, schooling, thnic groups, health inequalities e age group. Destacam-se prevalências de asma variando de $2,5 \%$ em alguns países do leste europeu 8 até $30 \%$ na Austrália 9 No Brasil, os estudos mostram pre- 
valências de asma e sintomas relacionados, em crianças, variando de 8,5\% 10 a 46,6\% 11 .

A revisão aponta ainda a expressiva utilização de medidas de auto-referência para asma 12,13. Em 2004, o Centers of Disease Control and Prevention (CDC, Estados Unidos) utilizou a referência de diagnóstico de asma em crianças relatada pelos pais para estimar sua prevalência nos Estados Unidos 13.

Desigualdade em saúde é um termo genérico utilizado para designar diferenças, variações e disparidades nas condições de saúde da população. As desigualdades em saúde assumem um papel importante no entendimento de asma, possivelmente influenciando na exposição a fatores de risco que favorecem o aparecimento da doença. A maioria das desigualdades em saúde entre grupos sociais, tais como classe social e raça/cor da pele, revela a distribuição de renda injusta e afeta os determinantes sociais da saúde 14 .

Recentes pesquisas na área de desigualdades em saúde e doenças respiratórias têm sido desenvolvidas, porém no Brasil pouco tem sido investigado a respeito do comportamento da asma segundo as diferentes regiões do país. Compreender melhor a dinâmica desse complexo processo pode contribuir para a implementação de políticas públicas dirigidas à promoção de saúde e eqüidade.

Os objetivos deste estudo foram estimar a prevalência de diagnóstico de asma auto-referida em crianças e analisá-la segundo as desigualdades sociais, demográficas e étnico-raciais no Brasil por meio dos dados da Pesquisa Nacional por Amostra de Domicílios (PNAD), 2003.

\section{Métodos}

Este estudo foi originado a partir de dados secundários referentes à população brasileira, oriundos da PNAD e seu suplemento sobre saúde, realizados em 2003.

O sistema de pesquisas domiciliares, implantado progressivamente no Brasil a partir de 1967, com a criação da PNAD, tem como finalidade a produção de informações básicas para o estudo do desenvolvimento socioeconômico do país. Trata-se de um sistema de pesquisas por amostra de domicílios que, por ter propósitos múltiplos, investiga diversas características socioeconômicas. O módulo sobre saúde vem sendo sistematicamente incluído a cada cinco anos desde 199815 .

A PNAD é realizada por meio de amostra probabilística de domicílios, em três estágios, a saber: municípios, setores censitários e domicílio.
No primeiro estágio, as unidades (municípios) foram classificadas em duas categorias: autorepresentativas (probabilidade 1 de pertencer à amostra) e não auto-representativas. Os municípios pertencentes à segunda categoria passaram por um processo de estratificação e, em cada estrato, foram selecionados com reposição e com probabilidade proporcional à população residente obtida no Censo Demográfico 2000. No segundo estágio, as unidades (setores censitários) foram selecionadas, em cada município da amostra, também com probabilidade proporcional e com reposição, sendo utilizado o número de unidades domiciliares existentes por ocasião do Censo Demográfico 2000 como medida de tamanho. No último estágio foram selecionados, com eqüiprobabilidade, em cada setor censitário da amostra, os domicílios particulares e as unidades de habitação em domicílios coletivos para investigação das características dos moradores e da habitação.

A PNAD foi realizada em 133.255 domicílios com 384.834 pessoas. O desenho amostral da pesquisa visou possibilitar a expansão dos seus resultados para o Brasil, grandes regiões, Unidades da Federação e nove regiões metropolitanas (Belém, Fortaleza, Recife, Salvador, Belo Horizonte, Rio de Janeiro, São Paulo, Curitiba e Porto Alegre).

\section{População de referência do estudo}

O presente estudo analisou os indivíduos na faixa etária infantil 16, entre 0 e 9 anos de idade, que participaram da PNAD 2003, totalizando 69.796 pessoas. A estimativa populacional para o ano de 2003 na faixa etária de investigação era de 34.340 .193 crianças, correspondendo a $19,41 \%$ da população brasileira (DATASUS. Sistema de Informações em Saúde. http://www.datasus.gov. br, acessado em 21/Abr/2008).

\section{Variável dependente}

Foi considerado como variável dependente o diagnóstico médico ou de outro profissional da saúde sobre asma/bronquite asmática na criança, auto-referido pelo responsável pelo domicílio. Esta pergunta está contida no grupo número 13 do questionário da $\mathrm{PNAD}$, referente às características de saúde dos moradores, e é descrita da seguinte forma "Algum médico ou profissional de saúde disse que (...) tem asma?”, e cujas possibilidades de resposta são "sim" ou "não", sugerindo tratar-se de prevalência diagnóstico de asma durante a vida. Para facilitar a compreensão dos entrevistados, evitando-se o não entendimento da pergunta, foi utilizada pelo Instituto Brasileiro de 
Geografia e Estatística (IBGE) a explicação sobre o termo asma como: "problema respiratório crônico, causado pela inflamação dos brônquios, que se caracteriza por crises de tosse e eliminação de catarro que duram pelo menos duas semanas, ou dificuldade para respirar, que se caracteriza por crises de falta de ar, produzindo ruído ou barulho sibilante no peito ou nas costas com som parecido com miados de gato..." 15 (p. 147).

\section{Variáveis independentes}

Foram consideradas como variáveis independentes as características demográficas (cor da pele do responsável e idade e sexo da criança), socioeconômicas (renda per capita, escolaridade do chefe do domicílio e localização geográfica da residência) e condições de moradia (tipo de material da casa, aglomeração no dormitório e combustível predominante utilizado no fogão do domicílio). Essas informações também foram obtidas por intermédio do responsável pelo domicílio.

A variável idade foi agrupada em menores de 2 anos; 3 a 7 e 8 a 9 anos. Cor da pele foi pesquisada conforme categorias estabelecidas pelo IBGE (branca, parda, preta, indígena, amarela) e auto-referida pelo responsável pela criança. Para análise dos dados foram excluídas as categorias indígena $(n=189)$ e amarela $(n=258)$ devido às baixas proporções na população do estudo $(0,27 \%$ e $0,37 \%$, respectivamente). Localização da residência foi categorizada em "urbana" (cidade ou vila em área urbanizada, cidade ou vila em área não urbanizada e área urbana isolada) e "rural" (aglomerado rural de extensão urbana, aglomerado rural isolado e povoado, aglomerado rural isolado - núcleo, aglomerado rural isolado outros aglomerados, e zona rural exclusive aglomerado rural). Renda per capita em Reais foi obtida usando-se a razão entre renda familiar total pelo número de pessoas residentes no domicílio e então dividida em quartis. Escolaridade do chefe do domicílio foi categorizada em anos de estudos com base nas categorias originais, a saber: "menos de 1 ano de estudo" (pré-escola, creche, alfabetização de adultos), "1 a 8 anos" (elementar - primário, médio 1o ciclo, ginasial, Ensino Fundamental ou primeiro grau), "9 a 11 anos" (médio segundo ciclo, científico e clássico, Ensino Médio ou segundo grau) e "12 ou mais anos de estudo" (superior e mestrado ou doutorado). O material das paredes externas do domicílio foi dicotomizado em "alvenaria" e "outros" (madeira aparelhada, palha, taipa revestida, madeira aproveitada e outro material). O combustível predominante utilizado no fogão foi dicotomizado em "gás" (gás canalizado e gás de botijão) e "lenha e carvão". A variável aglomeração foi obtida dividindo-se o número de cômodos utilizados como dormitórios pelo total de moradores do domicílio, e então, dividida em quartis.

A variável "ter consultado médico no último ano" foi utilizada apenas para a descrição da amostra.

\section{Análise estatística}

Os bancos de dados foram obtidos junto ao IBGE, com informações separadas sobre o domicílio e sobre os indivíduos. Criou-se um banco de dados com informações apenas sobre as crianças menores de 10 anos, no qual foram acrescentadas as informações sobre escolaridade e cor da pele do chefe da família, ambas oriundas do banco original. A análise estatística foi realizada utilizandose o programa estatístico Stata 9.0 (Stata Corp., College Station, Estados Unidos). Realizou-se a estatística descritiva sobre a prevalência de asma para o Brasil e regiões e as freqüências relativas e absolutas das variáveis de interesse do estudo, com os respectivos intervalos de 95\% de confiança (IC95\%). A análise dos fatores associados à asma foi realizada estratificada por região, sendo analisadas as regiões Sul e Nordeste por apresentarem, respectivamente, a mais alta e a mais baixa prevalências do desfecho investigado no Brasil.

Os fatores associados foram identificados usando-se o teste do qui-quadrado e de tendência linear quando oportuno. Todas as variáveis que nesses testes apresentaram $\mathrm{p}<0,25$ foram incluídas no modelo multivariável 17.

A análise multivariável foi realizada por meio da análise de regressão logística não condicional, obtendo-se uma estimativa de razão de chances (OR) e os respectivos IC95\% brutos e ajustados. Essa análise foi conduzida com base em um modelo teórico com abordagem hierarquizada ${ }^{18}$, no qual se assumiu que os fatores mais distais determinam o efeito dos níveis seguintes e assim, subseqüentemente, até a influência no desfecho. Esse tipo de análise proporciona o ajuste entre as variáveis de um mesmo nível e também por aquelas localizadas em níveis acima (Figura 1). Cor da pele do responsável e localização geográfica da residência encontram-se no primeiro nível, conseqüentemente influenciando os aspectos socioeconômicos (2o nível) e as condições de moradia (4o nível). Sexo e idade da criança condicionam o diagnóstico de asma.

Devido à complexidade do desenho amostral, foi utilizado o conjunto de comandos svy do programa estatístico Stata 9.0 a fim de considerar tanto o efeito do desenho como os pesos amostrais. 
Modelo teórico hierarquizado.

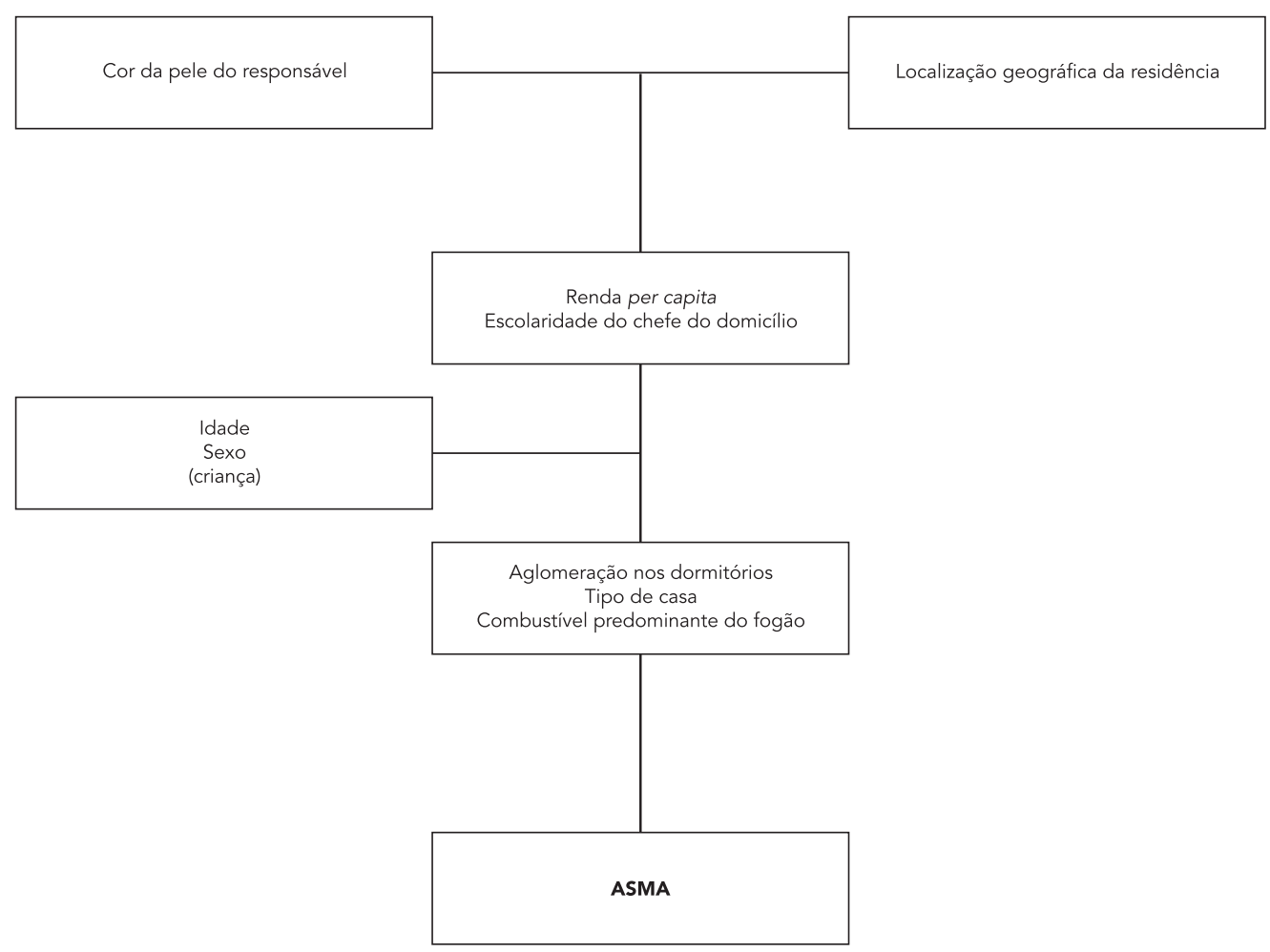

\section{Resultados}

Do total de crianças analisadas na PNAD, 32,3\% pertenciam à Região Nordeste, 13,7\% à Região Sul, e 39\%, 7,6\% e 7,4\% às regiões Sudeste, Norte e Centro-oeste, respectivamente. A renda média na Região Sul foi igual a R $\$ 291,71$ (desvio padrão: $\mathrm{R} \$ 443,98$ ) e o número médio de pessoas por dormitório foi igual a 2,35, enquanto no Nordeste observou-se renda per capita média igual a $\mathrm{R} \$$ 132,44 (DP: R\$ 256,75) e a aglomeração no dormitório igual a 2,67 (dados não apresentados).

A Figura 2 mostra as prevalências de diagnóstico de asma nas regiões do país e estratificadas segundo sexo. No Brasil, 8,1\% das crianças (IC95\%: 7,7-8,5) apresentaram asma, sendo a prevalência maior no Sul (12,6\%; IC95\%: 11,6-13,5) e menor no Nordeste (4,4\%; IC95\%: 4,0-4,8). Em todas as regiões, as prevalências de diagnóstico de asma foram maiores no sexo masculino.

A Tabela 1 apresenta a distribuição da amostra e a prevalência de diagnóstico de asma, segundo as variáveis independentes analisadas na Região
Sul. Nessa região, a maioria das crianças residia na zona urbana $(81,7 \%)$, apresentava idades entre 3 e 7 anos (52,1\%), 77,6\% de seus responsáveis auto-referiram ser de cor branca e $69,7 \%$ deles afirmaram ter até 8 anos de estudo. Observa-se que na Região Sul a prevalência de diagnóstico de asma foi maior entre crianças residentes na zona urbana (13,3\%; IC95\%: 12,3-14,3) e do sexo masculino (14,4\%; IC95\%: 13,2-15,7). Crianças cujos pais auto-referiram cor da pela preta $(21 \%$; IC95\%: 16,5-21,5) também apresentaram maior prevalência de diagnóstico de asma.

A distribuição da amostra e a prevalência de diagnóstico de asma, segundo as variáveis independentes analisadas na Região Nordeste, são apresentadas na Tabela 2. Nessa região, assim como na Região Sul, a maioria das crianças também residia na zona urbana $(67,4 \%)$, tinha idades entre 3 e 7 anos (51,8\%) e seus responsáveis apresentavam até 8 anos de estudo $(76,1 \%)$. Por outro lado, a maioria dos responsáveis pelas crianças auto-referiu cor da pele parda $(66,2 \%)$. A prevalência de diagnóstico de asma na Região 


\section{Figura 2}

Prevalência (\%) e intervalo de 95\% de confiança (IC95\%) de diagnóstico de asma em crianças de 0 a 9 anos, segundo regiões do país. Pesquisa Nacional por Amostra de Domicílios, 2003.

2a) Ambos os sexos

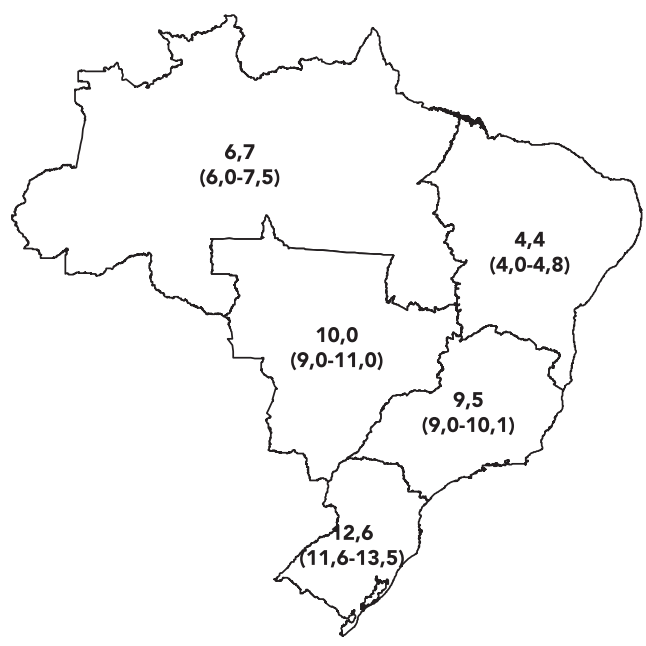

2b) Masculino

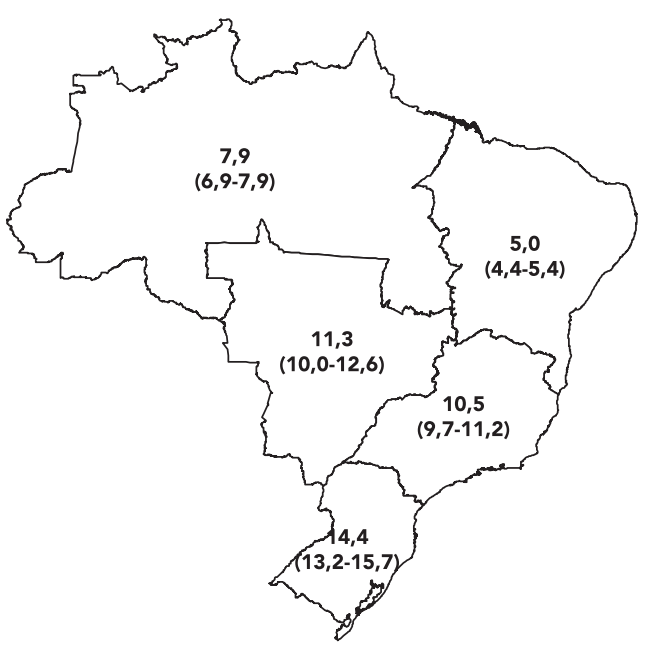

2c) Feminino

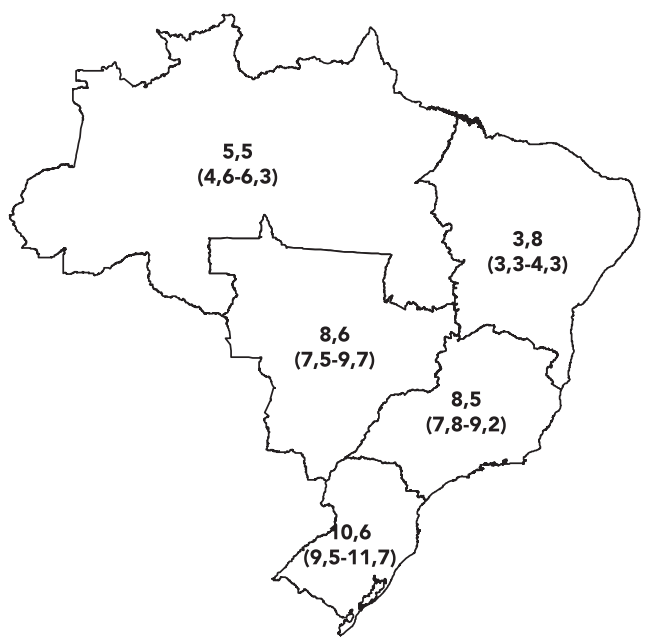

Nordeste foi maior entre as crianças residentes na zona urbana (5,1\%; IC95\%: 4,6-5,6), do sexo masculino (4,9\%; IC95\%: 4,4-5,4), com idades entre 3 e 7 anos (5,2\%; IC95\%: 4,7-5,7), que vivem em casas de alvenaria (4,6\%; IC95\%: 4,2-5,1) e com gás como combustível principal do fogão (4,7\%; IC95\%: 4,2-5,1) (Tabela 2).

Em ambas as regiões, mais de $60 \%$ das crianças consultaram médico no último ano. Tanto na
Região Sul (Tabela 1) quanto na Nordeste (Tabela 2), as prevalências maiores de diagnóstico de asma foram observadas dentre as crianças que visitaram o médico recentemente.

A Tabela 3 mostra as análises bruta e ajustada para os fatores associados ao diagnóstico de asma na Região Sul. Após análise ajustada, verificou-se que crianças cujos responsáveis auto-referiram cor da pele preta apresentaram uma chance $90 \%$ 
Tabela 1

Prevalência de diagnóstico de asma e distribuição da amostra, segundo as variáveis independentes analisadas em crianças de 0 a 9 anos de idade. Pesquisa Nacional por Amostra de Domicílios (PNAD), Região Sul, Brasil, 2003 ( $n=9.719$ ).

\begin{tabular}{|c|c|c|c|c|}
\hline \multirow[t]{2}{*}{ Variável } & \multicolumn{2}{|c|}{ Distribuição da amostra } & \multicolumn{2}{|c|}{ Asma/Bronquite } \\
\hline & $\mathrm{n}$ & $\%$ * & Prevalência * & IC95\% * \\
\hline \multicolumn{5}{|c|}{ Cor da pele do responsável [ $n=9.646]$} \\
\hline Branca & 7.540 & 77,6 & 12,0 & $11,0-13,0$ \\
\hline Parda & 1.450 & 16,8 & 12,5 & $10,7-14,4$ \\
\hline Preta & 656 & 5,6 & 21,0 & $16,5-25,5$ \\
\hline \multicolumn{5}{|l|}{ Local de residência [n = 9.719] } \\
\hline Zona urbana & 8.214 & 81,7 & 13,3 & $12,3-14,3$ \\
\hline Zona rural & 1.505 & 18,3 & 9,2 & $7,3-11,0$ \\
\hline \multicolumn{5}{|c|}{ Renda familiar per capita (Reais) [n = 9.633] } \\
\hline 320,50 ou mais & 2.406 & 24,5 & 10,7 & $9,3-12,10$ \\
\hline $175,25-320,00$ & 2.340 & 24,0 & 12,0 & $10,4-13,5$ \\
\hline $100,40-175,00$ & 2.297 & 24,3 & 13,5 & $11,5-15,5$ \\
\hline$<100,00$ & 2.590 & 27,2 & 14,1 & $12,3-15,9$ \\
\hline \multicolumn{5}{|c|}{ Escolaridade do chefe do domicílio (anos) [n = 8.876] } \\
\hline 12 ou mais & 689 & 7,4 & 11,1 & $8,6-13,7$ \\
\hline $9-11$ & 2.032 & 22,4 & 13,2 & $11,5-14,8$ \\
\hline $1-8$ & 6.112 & 69,7 & 12,5 & $11,4-13,6$ \\
\hline$<1$ & 43 & 0,5 & 15,2 & $4,7-25,6$ \\
\hline \multicolumn{5}{|l|}{ Sexo $[n=9.719]$} \\
\hline Masculino & 4.939 & 50,5 & 14,4 & $13,2-15,7$ \\
\hline Feminino & 4.780 & 49,5 & 10,6 & $9,5-11,7$ \\
\hline \multicolumn{5}{|l|}{ Idade (anos) $[\mathrm{n}=9.719]$} \\
\hline $0-2$ & 2.503 & 25,5 & 12,5 & $11,0-14,1$ \\
\hline $3-7$ & 5.070 & 52,1 & 13,6 & $12,4-14,7$ \\
\hline $8-9$ & 2.146 & 22,4 & 10,1 & $8,7-11,6$ \\
\hline \multicolumn{5}{|c|}{ Aglomeração (pessoas/dormitório - quartis) [n = 9.717] } \\
\hline$\leq 1,50$ & 2.490 & 25,8 & 11,1 & $9,8-12,4$ \\
\hline $1,60-2,00$ & 3.242 & 34,1 & 11,6 & $10,3-12,9$ \\
\hline $2,20-3,00$ & 2.688 & 26,9 & 13,4 & $11,8-15,0$ \\
\hline $3,25-12,00$ & 1.297 & 13,2 & 16,1 & $13,4-18,7$ \\
\hline \multicolumn{5}{|l|}{ Tipo de casa $[n=9.717]$} \\
\hline Alvenaria & 6.174 & 62,3 & 13,2 & $12,2-14,3$ \\
\hline Madeira/Palha/Taipa/Outros & 3.543 & 37,7 & 11,4 & $9,9-12,9$ \\
\hline \multicolumn{5}{|c|}{ Combustível predominante do fogão [n = 9.702] } \\
\hline Gás & 8.653 & 86,3 & 12,9 & $12,0-13,9$ \\
\hline Lenha/Carvão & 1.049 & 13,7 & 10,0 & $7,5-12,5$ \\
\hline \multicolumn{5}{|c|}{ Consulta com médico(a) no último ano [n = 9.715] } \\
\hline Não & 2.872 & 30,9 & 6,0 & $5,0-7,0$ \\
\hline Sim & 6.843 & 69,1 & 15,5 & $14,3-16,6$ \\
\hline Total & 9.719 & 100,0 & 12,5 & $11,6-13,5$ \\
\hline
\end{tabular}

* Ajustados pelos pesos amostrais e pelo efeito da amostra por conglomerados. 
Tabela 2

Prevalência de diagnóstico de asma e distribuição da amostra, segundo as variáveis independentes analisadas em crianças de 0 a 9 anos de idade. Pesquisa Nacional por Amostra de Domicílios (PNAD), Região Nordeste, Brasil, 2003 ( $n=24.624$ ).

\begin{tabular}{|c|c|c|c|c|}
\hline \multirow[t]{2}{*}{ Variável } & \multicolumn{2}{|c|}{ Distribuição da amostra } & \multicolumn{2}{|c|}{ Asma/Bronquite } \\
\hline & $\mathrm{n}$ & $\%$ * & Prevalência * & IC95\% * \\
\hline \multicolumn{5}{|c|}{ Cor da pele do responsável [ $n=24.531]$} \\
\hline Branca & 6.402 & 25,8 & 4,9 & $4,2-5,6$ \\
\hline Parda & 15.863 & 66,2 & 4,2 & $3,8-4,7$ \\
\hline Preta & 2.266 & 8,0 & 4,2 & $3,1-5,3$ \\
\hline \multicolumn{5}{|l|}{ Local de residência $[n=24.624]$} \\
\hline Zona urbana & 18.061 & 67,4 & 5,1 & $4,6-5,6$ \\
\hline Zona rural & 6.563 & 32,6 & 3,0 & $2,4-3,6$ \\
\hline \multicolumn{5}{|c|}{ Renda familiar per capita (Reais) [ $n=24.266]$} \\
\hline 130,20 ou mais & 6.031 & 21,9 & 5,0 & $4,3-5,7$ \\
\hline $72,50-130,00$ & 6.086 & 24,6 & 4,1 & $3,5-4,7$ \\
\hline $41,25-72,40$ & 6.053 & 26,2 & 4,5 & $3,8-5,3$ \\
\hline$<41,25$ & 6.096 & 27,3 & 4,1 & $3,5-4,8$ \\
\hline \multicolumn{5}{|c|}{ Escolaridade do chefe do domicílio (anos) [n =19.143] } \\
\hline 12 ou mais & 690 & 3,0 & 6,7 & $4,7-8,7$ \\
\hline $9-11$ & 3.474 & 15,9 & 5,7 & $4,7-6,7$ \\
\hline $1-8$ & 14.153 & 76,1 & 4,5 & $4,0-5,0$ \\
\hline$<1$ & 826 & 5,0 & 3,3 & $2,0-4,6$ \\
\hline \multicolumn{5}{|l|}{ Sexo $[n=24.624]$} \\
\hline Masculino & 12.474 & 50,6 & 4,9 & $4,4-5,4$ \\
\hline Feminino & 12.150 & 49,4 & 3,8 & $3,4-4,3$ \\
\hline \multicolumn{5}{|l|}{ Idade (anos) $[\mathrm{n}=24.624]$} \\
\hline $0-2$ & 6.264 & 26,8 & 3,3 & $2,8-3,8$ \\
\hline $3-7$ & 12.690 & 51,8 & 5,2 & $4,7-5,7$ \\
\hline $8-9$ & 5.310 & 21,4 & 3,8 & $3,2-4,4$ \\
\hline \multicolumn{5}{|c|}{ Aglomeração (pessoas/dormitório - quartis) [n = 24.578] } \\
\hline$\leq 2$ & 10.240 & 40,6 & 4,7 & $4,1-5,2$ \\
\hline $2,16-2,50$ & 4.416 & 18,9 & 4,5 & $3,7-5,3$ \\
\hline $2,60-3,00$ & 4.701 & 19,2 & 3,7 & $3,1-4,3$ \\
\hline $3,25-12,00$ & 5.221 & 21,3 & 4,4 & $3,6-5,2$ \\
\hline \multicolumn{5}{|l|}{ Tipo de casa $[n=24.578]$} \\
\hline Alvenaria & 22.498 & 89,2 & 4,6 & $4,2-5,0$ \\
\hline Madeira/Palha/Taipa/Outros & 2.080 & 10,8 & 3,0 & $2,2-3,8$ \\
\hline \multicolumn{5}{|c|}{ Combustivel predominante do fogão [n = 23.996] } \\
\hline Gás & 19.047 & 75,2 & 4,7 & $4,2-5,1$ \\
\hline Lenha/Carvão & 4.949 & 24,8 & 3,4 & $2,7-4,1$ \\
\hline \multicolumn{5}{|c|}{ Consulta com médico(a) no último ano [n = 24.620] } \\
\hline Não & 8.524 & 37,6 & 1,9 & $1,5-2,3$ \\
\hline Sim & 16.096 & 62,4 & 5,9 & $5,3-6,5$ \\
\hline Total & 24.624 & 100,0 & 4,4 & $4,0-4,8$ \\
\hline
\end{tabular}

* Ajustados pelos pesos amostrais e pelo efeito da amostra por conglomerados. 
Tabela 3

Fatores associados ao diagnóstico de asma em crianças com idades entre 0 e 9 anos. Análise de regressão logística bruta e ajustada. Pesquisa Nacional por Amostra de Domicílios (PNAD), Região Sul, Brasil, 2003.

\begin{tabular}{|c|c|c|c|c|c|}
\hline \multirow[t]{2}{*}{ Nível } & \multirow[t]{2}{*}{ Variável } & \multicolumn{2}{|c|}{ Análise bruta } & \multicolumn{2}{|c|}{ Análise ajustada } \\
\hline & & OR (IC95\%) & Valor de $p$ & OR (IC95\%) & Valor de $p$ * \\
\hline \multirow[t]{7}{*}{1} & Cor da pele do responsável & & $<0,001 * \star$ & & $<0,001 * * *$ \\
\hline & Branca & 1,0 & & 1,0 & \\
\hline & Parda & $1,1(0,9-1,3)$ & & $1,1(0,9-1,3)$ & \\
\hline & Preta & $1,9(1,5-2,6)$ & & $1,9(1,4-2,5)$ & \\
\hline & Local de residência & & $<0,001 * \star$ & & $<0,001 * * *$ \\
\hline & Zona urbana & 1,0 & & 1,0 & \\
\hline & Zona rural & $0,7(0,5-0,8)$ & & $0,7(0,5-0,8)$ & \\
\hline \multirow[t]{10}{*}{2} & Renda familiar per capita (Reais) & & $<0,001 \#$ & & $0,001 \# \#$ \\
\hline & 320,50 ou mais & 1,0 & & 1,0 & \\
\hline & $175,25-320,00$ & $1,1(0,9-1,4)$ & & $1,2(1,0-1,5)$ & \\
\hline & $100,40-175,00$ & $1,3(1,1-1,6)$ & & $1,5(1,2-1,8)$ & \\
\hline & $<100,00$ & $1,4(1,1-1,7)$ & & $1,5(1,1-1,8)$ & \\
\hline & Escolaridade do chefe do domicílio (anos) & & 0,203 \# & & $0,468 \# \#$ \\
\hline & 12 ou mais & 1,0 & & 1,0 & \\
\hline & $9-11$ & $1,2(0,9-1,6)$ & & $1,0(0,8-1,4)$ & \\
\hline & $1-8$ & $1,1(0,9-1,5)$ & & $0,9(0,7-1,3)$ & \\
\hline & $<1$ & $1,4(0,6-3,1)$ & & $1,2(0,5-3,1)$ & \\
\hline \multirow[t]{7}{*}{3} & Sexo & & $<0,001 * *$ & & $<0,001 \# \#$ \\
\hline & Masculino & 1,0 & & 1,0 & \\
\hline & Feminino & $0,7(0,6-0,8)$ & & $0,7(0,6-0,8)$ & \\
\hline & Idade (anos) & & 0,010 \# & & 0,045 \#\#\# \\
\hline & $0-2$ & 1,0 & & 1,0 & \\
\hline & $3-7$ & $1,1(0,9-1,3)$ & & $1,1(1,0-1,3)$ & \\
\hline & $8-9$ & $0,8(0,7-1,0)$ & & $0,8(0,7-1,0)$ & \\
\hline \multirow[t]{11}{*}{4} & Aglomeração (pessoas/dormitório - quartis) & & $<0,001 \#$ & & $0,037 \S$ \\
\hline & 1,50 ou menos & 1,0 & & 1,0 & \\
\hline & $1,60-2,00$ & $1,1(0,9-1,3)$ & & $1,0(0,8-1,2)$ & \\
\hline & $2,20-3,00$ & $1,2(1,0-1,5)$ & & $1,1(0,9-1,3)$ & \\
\hline & $3,25-12,00$ & $1,5(1,2-1,9)$ & & $1,3(1,0-1,6)$ & \\
\hline & Tipo de casa & & 0,052 ** & & $0,031 \S$ \\
\hline & Alvenaria & 1,0 & & 1,0 & \\
\hline & Madeira/Palha/Taipa/Outros & $0,8(0,7-1,0)$ & & $0,8(0,7-1,0)$ & \\
\hline & Combustível predominante do fogão & & $0,043 * *$ & & $0,344 \S$ \\
\hline & Gás & 1,0 & & 1,0 & \\
\hline & Lenha/Carvão & $0,8(0,6-1,0)$ & & $0,9(0,6-1,2)$ & \\
\hline
\end{tabular}

* Valor de $\mathrm{p}$ de heterogeneidade de Wald;

** Valor de $p$ do teste do qui-quadrado;

*** Ajustado pelas variáveis do mesmo nível;

\# Valor de p do teste de tendência linear;

\#\# Ajustado pelas variáveis do nível 1 + variáveis do mesmo nível;

\#\#\# Ajustado pelas variáveis do nível 1 + renda per capita + variáveis do mesmo nível;

$\S$ Ajustado pelas variáveis do nível 1 + renda per capita + variáveis do nível 3 + variáveis do mesmo nível. 
maior de diagnóstico de asma quando comparadas às crianças com responsáveis de cor da pele branca. Quanto menor a renda mensal per capita, maior a chance do desfecho, o mesmo ocorrendo com crianças entre 3 e 7 anos de idade quando comparadas às com idades entre 0 e 2 anos. Adicionalmente, apresentar mais do que três pessoas por cômodo considerado dormitório também mostrou-se positivamente associado ao diagnóstico de asma. Por outro lado, residir em zona rural, ser do sexo feminino e morar em casas cujas paredes foram feitas de madeira, palha, taipa ou outros materiais foram associados negativamente ao desfecho.

Na Tabela 4, verifica-se as análises bruta e ajustada para fatores associados à asma na Região Nordeste. Observa-se após o ajuste, uma maior chance de diagnóstico de asma dentre as famílias com renda per capita inferior a $\mathrm{R} \$ 41,25$, as crianças de 3 a 7 anos quando comparadas às de 0 a 2 anos. Crianças residentes na zona rural, do sexo feminino e com responsáveis com escolaridade inferior a 1 ano, quando comparados ao grupo com 12 anos ou mais de estudos, apresentaram-se associadas negativamente à presença do desfecho.

\section{Discussão}

O presente estudo teve como objetivo comparar as desigualdades sociais, demográficas e étnicoraciais relacionadas ao diagnóstico de asma em crianças menores de 10 anos de idade nas regiões Sul e Nordeste do Brasil, reconhecidamente distintas em termos culturais, sociais, econômicos e de condições climáticas. Os resultados obtidos apontam para desigualdades mais evidentes na Região Sul.

A prevalência de asma observada no Brasil $(8,1 \%)$ foi ligeiramente menor quando comparada àquelas encontradas em estudos realizados no Egito $(9,4 \%) 19$ e na Itália $(11,7 \%) 20$, porém, muito menores do que as encontradas em outras regiões como em cidades da Austrália (27,3\%) 9 . Na Região Sul, a prevalência do desfecho foi semelhante aos 12,8\% encontrados em Pelotas, Rio Grande do Sul 4, porém ligeiramente menor do que as prevalências encontradas em Santa Maria, Rio Grande do Sul (14,9\%) 21, e maior do que em Itajaí, Santa Catarina, (10,3\%) 22. A região Nordeste apresentou prevalência de asma menor do que as encontradas em Recife, Pernambuco, em 2002 (14,8\%) 23, Natal, Rio Grande do Norte $(16,1 \%) 22$ e Vitória da Conquista, Bahia $(11,5 \%) 22$. As diferenças encontradas nas prevalências podem ser atribuídas, pelo menos em parte, às diversas formas de mensuração do desfecho 24. Nos estudos das cidades do Nordeste foram utilizados como desfechos os sintomas de asma e não o diagnóstico médico de asma. A proporção de crianças que consultaram médico no último ano foi semelhante nas duas regiões estudadas. Entretanto, ao se considerar a vida toda da criança, possivelmente o acesso ao médico na Região Nordeste será menor, podendo ser um dos responsáveis por maior subnotificação da doença ao longo da vida destas crianças, quando comparada à Região Sul. Relatório da OMS aponta que metade dos médicos do Brasil está concentrada em $30 \%$ da população residente nas regiões com menor taxa de pobreza 25 .

Crianças sofrem muitas mudanças hormonais, comportamentais, dentre outras, até a idade adulta. Essas mudanças devem ser consideradas para o entendimento de exposições de risco para crianças 26. Nossos resultados apontam para uma maior prevalência na idade entre 3 e 7 anos, tanto na Região Nordeste quanto na Região Sul, semelhante aos resultados obtidos por Roberts 27, nos Estados Unidos. Um estudo realizado na Escócia 28 , revela que o pico de prevalência de asma ocorre entre os 4 e 6 anos de idade, podendo ser explicado como reflexo da entrada na escola, onde as crianças são expostas a mais infecções respiratórias, resultando em maior procura por consultas médicas, o que pode aumentar a prevalência de diagnóstico médico de asma. Menor chance de diagnóstico de asma dentre as meninas até os 10 anos de idade é corroborada por outros estudos como, por exemplo, um artigo de revisão 29 e outros realizados nos Estados Unidos 30,31,32,33 e em São Paulo 34 . Na adolescência há uma inversão das prevalências entre os sexos 23,35 , podendo ser explicada, em parte, por diferentes percepções da doença e por mudanças nos comportamentos relacionados à saúde durante e após a puberdade 28. Diferenças anatômicas entre os aparelhos respiratórios de crianças também são apontadas como responsáveis pelas divergências na prevalência de asma entre os sexos 36 .

Crianças residentes na zona urbana tiveram maior chance de diagnóstico de asma do que as residentes na zona rural, em ambas as regiões estudadas, corroborando outras pesquisas 35,37 . Essas diferenças podem ser explicadas por fatores sabidamente associados à asma e que estão mais presentes nas localidades urbanas, tais como exposição ao tabaco, poluição, prematuridade, casas de baixo padrão e maus hábitos alimentares 37 . Residentes em zonas urbanas têm mais facilidade de acesso a serviços de saúde. Como o desfecho foi baseado na presença de diagnóstico por profissional de saúde de asma, podemos supor que a subnotificação de diagnóstico 
Tabela 4

Fatores associados ao diagnóstico de asma em crianças com idades entre 0 e 9 anos. Análise de regressão logística bruta e ajustada. Pesquisa Nacional por Amostra de Domicílios (PNAD), Região Nordeste, Brasil, 2003.

\begin{tabular}{|c|c|c|c|c|c|}
\hline \multirow[t]{2}{*}{ Nível } & \multirow[t]{2}{*}{ Variável } & \multicolumn{2}{|c|}{ Análise bruta } & \multicolumn{2}{|c|}{ Análise ajustada } \\
\hline & & OR (IC95\%) & Valor de $p$ & OR (IC95\%) & Valor de $p$ * \\
\hline \multirow[t]{7}{*}{1} & Cor da pele do responsável & & $0,181 * *$ & & $0,141 * \star \star$ \\
\hline & Branca & 1,0 & & 1,0 & \\
\hline & Parda & $0,9(0,7-1,0)$ & & $0,9(0,8-1,0)$ & \\
\hline & Preta & $0,9(0,6-1,1)$ & & $0,8(0,6-1,1)$ & \\
\hline & Local de residência & & $<0,001 * \star$ & & $<0,001 * * *$ \\
\hline & Zona urbana & 1,0 & & 1,0 & \\
\hline & Zona rural & $0,6(0,5-0,7)$ & & $0,6(0,5-0,7)$ & \\
\hline \multirow[t]{10}{*}{2} & Renda familiar per capita (Reais) & & 0,084 \# & & $0,031 \# \#$ \\
\hline & 130,20 ou mais & 1,0 & & 1,0 & \\
\hline & $72,50-130,00$ & $0,8(0,7-1,0)$ & & $0,9(0,7-1,1)$ & \\
\hline & $41,25-72,40$ & $0,9(0,7-1,1)$ & & $1,1(0,9-1,4)$ & \\
\hline & $<41,25$ & $0,8(0,7-1,0)$ & & $1,3(1,0-1,6)$ & \\
\hline & Escolaridade do chefe do domicílio (anos) & & $<0,001 \#$ & & $0,003 \# \#$ \\
\hline & 12 ou mais & 1,0 & & 1,0 & \\
\hline & $9-11$ & $0,8(0,6-1,2)$ & & $0,8(0,6-1,2)$ & \\
\hline & $1-8$ & $0,6(0,5-0,9)$ & & $0,7(0,5-1,0)$ & \\
\hline & $<1$ & $0,5(0,3-0,8)$ & & $0,5(0,3-0,9)$ & \\
\hline \multirow[t]{7}{*}{3} & Sexo & & $<0,001 * \star$ & & $<0,001 \# \#$ \\
\hline & Masculino & 1,0 & & 1,0 & \\
\hline & Feminino & $0,8(0,7-0,9)$ & & $0,7(0,7-0,9)$ & \\
\hline & Idade (anos) & & $0,096 \#$ & & 0,027 \#\# \\
\hline & $0-2$ & 1,0 & & 1,0 & \\
\hline & $3-7$ & $1,6(1,4-1,9)$ & & $1,6(1,3-1,9)$ & \\
\hline & $8-9$ & $1,2(0,9-1,4)$ & & $1,2(1,0-1,5)$ & \\
\hline \multirow[t]{11}{*}{4} & Aglomeração (pessoas/dormitório - quartis) & & $<0,196 \#$ & & $0,653 \S$ \\
\hline & 2 ou menos & 1,0 & & 1,0 & \\
\hline & $2,16-2,50$ & $1,0(0,8-1,2)$ & & $1,0(0,8-1,3)$ & \\
\hline & $2,60-3,00$ & $0,8(0,6-1,0)$ & & $0,9(0,7-1,1)$ & \\
\hline & $3,25-12,00$ & $1,0(0,8-1,2)$ & & $1,0(0,8-1,3)$ & \\
\hline & Tipo de casa & & 0,002 ** & & $0,139 \S$ \\
\hline & Alvenaria & 1,0 & & 1,0 & \\
\hline & Madeira/Palha/Taipa/Outros & $0,6(0,5-0,8)$ & & $0,8(0,5-1,1)$ & \\
\hline & Combustível predominante do fogão & & $0,004 * *$ & & $0,836 \S$ \\
\hline & Gás & 1,0 & & 1,0 & \\
\hline & Lenha/Carvão & $0,7(0,6-0,9)$ & & $1,0(0,8-1,4)$ & \\
\hline
\end{tabular}

* Valor de $\mathrm{p}$ de heterogeneidade de Wald;

** Valor de $p$ do teste do qui-quadrado;

*** Ajustado pelas variáveis do mesmo nível;

\# Valor de p do teste de tendência linear;

\#\# Ajustado pelas variáveis do nível 1 + variáveis do mesmo nível;

\#\#\# Ajustado pelas variáveis do nível 1 + renda per capita + variáveis do mesmo nível;

$\S$ Ajustado pelas variáveis do nível 1 + renda per capita + variáveis do nível 3 + variáveis do mesmo nível. 
também pode ter contribuído para as diferenças observadas.

Cor da pele preta associada à maior chance de diagnóstico de asma foi observada em outros estudos, corroborando nosso resultado $4,27,32,38,39,40,41$. Chor \& Lima 42 alegam que mesmo não sendo útil como categoria biológica, a raça é um determinante de identidades, acesso a recursos e valorização da sociedade, que parecem estar mais presentes na Região Sul em comparação à Região Nordeste. Processos discriminatórios institucionalizados 43 levam minorias étnicas a terem menos acesso a bens, oportunidades e serviços de saúde, aumentando a chance de asma. Esses processos podem ser diferentes entre as regiões analisadas, necessitando de outros tipos de estudos para melhor esclarecimento a respeito.

Baixa escolaridade do responsável foi associada com menor chance de asma no Nordeste, corroborado por estudos realizados na Colômbia 44 e Israel 35 . Por outro lado, outras pesquisas realizadas na Turquia 45, nos Estados Unidos 46 e na República Tcheca 47 apontam para direção contrária. No Brasil, pesquisa com variáveis agregadas corrobora nossos resultados 5 , enquanto um estudo com variáveis de nível individual não encontrou associação entre níveis educacionais e asma 4. É possível supor que pais com menor nível de escolaridade compreendem menos os problemas de saúde, procuram menos pelos serviços de saúde, podendo levar a um subdiagnóstico de asma nestas populações 19,23. Rendas per capita baixas apresentaram-se como risco para asma em ambas as regiões, corroborando os resultados obtidos na Geórgia 46 e em Nova Iorque 30 (Estados Unidos). Resultados de estudos brasileiros, realizados em Pelotas, não evidenciaram a associação da renda com prevalência de asma ${ }^{4}$ ou com atendimentos de emergência desencadeados pela doença ${ }^{48}$. Renda é uma medida muito útil de posição socioeconômica devido à sua direta relação com condições materiais que podem influenciar na saúde das pessoas 49 . Assim, é plausível que muitos dos efeitos da renda sobre a saúde dos indivíduos podem ser entendidos pela forma na qual o dinheiro e os bens são convertidos em gastos que provêem ambientes favoráveis à saúde, incluindo alimentação adequada e acesso a serviços 50 .

A aglomeração nos espaços utilizados como dormitórios esteve positivamente associada à asma, indo ao encontro de outras pesquisas 51,52 . A aglomeração pode influenciar no aumento de doenças respiratórias, principalmente com infecções cruzadas entre os moradores ${ }^{53}$. Temperaturas mais baixas no Sul proporcionam ambientes fechados por períodos mais longos, diminuindo a circulação de ar, facilitando essas infecções e também os processos alérgicos. Estudo feito por Gonzáles et al. 54 com a fumaça de cigarro, identificou a relação positiva de lugares com menor circulação de ar e asma. Residir em casas cujas paredes eram de madeira, palha, taipa ou outros materiais se apresentou como um fator de proteção para o diagnóstico de asma na Região Sul.

Nossos resultados sugerem que as desigualdades em saúde relacionadas à asma em crianças são mais evidentes na Região Sul. Apontase principalmente as diferenças observadas nas condições de moradia dentre ambas as regiões. Estudo sobre desigualdades em saúde segundo as regiões brasileiras 25 também aponta indicadores mais desfavoráveis nas regiões Norte e Nordeste, quando comparados às demais regiões.

A utilização de dados secundários é importante na área da saúde, pois informações desta amplitude podem proporcionar o entendimento de determinados agravos, sua magnitude e distribuição, bem como auxiliar no planejamento de políticas públicas em saúde. É importante salientar que níveis de morbidade em crianças podem ser prevenidos, desde que existam, também, mudanças em aspectos demográficos, socioeconômicos, entre outros, que podem interagir com as ações em Saúde Pública 55. Em inquéritos de saúde, como é o caso da PNAD, rotineiramente são coletadas informações sobre morbidade de forma auto-referida. Essa metodologia tem sido cada vez mais empregada nos países desenvolvidos 13 devido aos bons níveis de concordância, reprodutibilidade e custo-benefício quando se consideram os resultados obtidos de avaliações clínicas 56,57. Por outro lado, estudos com dados secundários podem apresentar algumas limitações, como a indisponibilidade de outras informações. Por exemplo, na PNAD 2003, informações sobre tabagismo não foram coletadas, sendo este um fator adicional que contribuiria para melhor identificar as diferenças regionais analisadas. Adicionalmente, a pesquisa da PNAD é de caráter transversal, o que não nos permite inferir causalidade. $\mathrm{O}$ viés de memória também pode estar presente, pois tanto o desfecho quanto as exposições foram medidas retrospectivamente.

Nossos resultados podem encorajar a formulação de novas políticas visando à diminuição dos riscos associados à asma. Pesquisas avaliando informações de nível agregado, além de abordagens qualitativas, também poderiam ser realizadas com o intuito de melhorar a compreensão da dinâmica da doença e o entendimento das desigualdades associadas à asma. 


\section{Resumo}

Estimou-se a prevalência de diagnóstico de asma em crianças e as desigualdades sociodemográficas entre as regiões Sul e Nordeste do Brasil. Analisou-se os dados de menores de 10 anos de idade da Pesquisa Nacional de Amostra por Domicílios, $2003(n=69.796)$. Características socioeconômicas, sexo e idade da criança e cor da pele do responsável foram as variáveis independentes analisadas, por meio de regressão logística não condicional. A prevalência de diagnóstico de asma no Brasil foi de 8,1\% (IC95\%: 7,7-8,5), sendo de 12,6\% (IC95\%: 11,6-13,5) e 4,4\% (IC95\%: 4,0-4,8) no Sul e Nordeste, respectivamente. Crianças entre 3 e 7 anos de idade e as de família com menor renda apresentaram maiores chances de diagnóstico de asma em ambas as regiões. Ser filho de pais que auto-referiram cor da pele preta, viver em aglomeração e residir em casas de baixo padrão foram associados positivamente a diagnóstico de asma no Sul. No Nordeste, menor escolaridade dos responsáveis foi associada a menores chances de diagnóstico de asma. As desigualdades foram mais evidentes na Região Sul, sugerindo variações no processo de determinação e entendimento da doença no país.

Asma; Desigualdades em Saúde; Criança

\section{Colaboradores}

F. C. Wehrmeister construiu o banco de dados, fez a análise estatística e redigiu o artigo. K. G. A. Peres orientou a análise estatística e realizou a revisão crítica do artigo.

\section{Referências}

1. Chiesa AM, Westphal MF, Akerman M. Doenças respiratórias agudas: um estudo das desigualdades em saúde. Cad Saúde Pública 2008; 24:55-69.

2. Muiño A, Menezes AMB, Reichert FF, Duquia RP, Chatkin M. Wheezing phenotypes from birth to adolescence: a cohort study in Pelotas, Brazil, 1993-2004. J Bras Pneumol 2007; 34:347-55.

3. Asher MI, Montefort S, Bjorksten B, Lai CKW, Strachan DP, Weiland SK, et al. Worldwide time trends in the prevalence of symptoms of asthma, allergic rhinoconjunctivitis, and eczema in childhood: ISAAC Phases One and Three repeat multicountry cross-sectional surveys. Lancet 2006; 368:733.

4. Chatkin MN, Menezes AM. Prevalence and risk factors for asthma in schoolchildren in southern Brazil. J Pediatr (Rio J.) 2005; 81:411-6.

5. Cunha SS, Pujades-Rodriguez M, Barreto ML, Genser B, Rodrigues LC. Ecological study of socioeconomic indicators and prevalence of asthma in schoolchildren in urban Brazil. BMC Public Health 2007; 7:205.
6. Redd SC. Asthma in the United States: burden and current theories. Environ Health Perspect 2002; 110 Suppl 4:557-60.

7. Redd SC, Mokdad AH. Invited commentary: obesity and asthma: new perspectives, research needs, and implications for control programs. Am J Epidemiol 2002; 155:198-202.

8. Masoli M, Fabian D, Holt S, Beasley R. Global burden of asthma. Wellington: Medical Research Institute of New Zealand/Southampton: University of Southampton; s.d.

9. Haby MM, Peat JK, Marks GB, Woolcock AJ, Leeder SR. Asthma in preschool children: prevalence and risk factors. Thorax 2001; 56:589-95.

10. Barros MBA, César CLG, Carandina L, Torre GD. Desigualdades sociais na prevalência de doenças crônicas no Brasil, PNAD-2003. Ciênc Saúde Coletiva 2006; 11:911-26. 
11. Boechat JL, Rios JL, Sant'anna CC, França AT. Prevalência e gravidade de sintomas relacionados à asma em escolares e adolescentes no Município de Duque de Caxias, Rio de Janeiro. J Bras Pneumol 2005; 31:111-7.

12. Pekkanen J, Pearce N. Defining asthma in epidemiological studies. Eur Respir J 1999; 14:951-7.

13. Centers for Disease Control and Prevention. Asthma prevalence and control characteristics by race/ ethnicity: United States, 2002. MMWR Morb Mortal Wkly Rep 2004; 53:145-8.

14. Organização Pan-Americana da Saúde. Saúde nas Américas. Washington DC: Organização Pan-Americana da Saúde; 2007.

15. Instituto Brasileiro de Geografia e Estatística. Pesquisa Nacional por Amostra de Domicílio: acesso e utilização de serviços, 2003. Rio de Janeiro: Instituto Brasileiro de Geografia e Estatística; 2005.

16. Reis JN, Martin CCS, Ferriani MGC. Mulheres vítimas de abuso sexual: meios coercitivos e produção de lesões não genitais. Cad Saúde Pública 2004; 20:465-73.

17. Hosmer DW, Lemeshow S. Applied logistic regression. New York: John Wiley \& Sons; 1989.

18. Victora CG, Huttly SR, Fuchs SC, Olinto MT. The role of conceptual frameworks in epidemiological analysis: a hierarchical approach. Int J Epidemiol 1997; 26:224-7.

19. Georgy V, Fahim HI, El-Gaafary M, Walters S. Prevalence and socioeconomic associations of asthma and allergic rhinitis in northern Africa. Eur Respir J 2006; 28:756-62.

20. Cesaroni G, Farchi S, Davoli M, Forastiere F, Perucci CA. Individual and area-based indicators of socioeconomic status and childhood asthma. Eur Respir J 2003; 22:619-24.

21. Cassol VE, Solé D, Mena-Barreto SS, Teche SP, Rizzato TM, Maldonado M, et al. Prevalence of asthma among adolescents in the city of Santa Maria, in the state of Rio Grande do Sul, Brazil. International Study of Asthma and Allergies in Childhood (ISAAC) Project. J Bras Pneumol 2005; 31:191-6.

22. Sole D, Wandalsen GF, Camelo-Nunes IC, Naspitz CK. Prevalence of symptoms of asthma, rhinitis, and atopic eczema among Brazilian children and adolescents identified by the International Study of Asthma and Allergies in Childhood (ISAAC) Phase 3. J Pediatr (Rio J.) 2006; 82:341-6.

23. Britto MCA, Bezerra PGM, Brito RCCM, Rego JC, Burity EF, Alves JGB. Asthma in schoolchildren from Recife, Brazil. Prevalence comparison: 199495 and 2002. J Pediatr (Rio J.) 2004; 80:391-400.

24. Felizola MLBM, Viegas CAA, Almeida M, Ferreira F, Santos MCA. Prevalência de asma brônquica e de sintomas a ela relacionados em escolares do Distrito Federal e sua relação com o nível socioeconômico. J Bras Pneumol 2005; 31:486-91.

25. Duarte EC, Schneider MC, Paes-Sousa R, Ramalho WM, Sardinha LMV, Silva Júnior JB, et al. Epidemiologia das desigualdades em saúde no Brasil. Um estudo exploratório. Brasília: Organização Pan-Americana da Saúde; 2002.

26. Thompson KM. Changes in children's exposure as a function of age and the relevance of age definitions for exposure and health risk assessment. MedGenMed 2004; 6:2.
27. Roberts EM. Does your child have asthma? Parent reports and medication use for pediatric asthma. Arch Pediatr Adolesc Med 2003; 157:449-55.

28. Osman M, Hansell AL, Simpson CR, Hollowell J, Helms PJ. Gender-specific presentations for asthma, allergic rhinitis and eczema in primary care. Prim Care Respir J 2007; 16:28-35.

29. Almqvist C, Worm M, Leynaert B. Impact of gender on asthma in childhood and adolescence: a GA2LEN review. Allergy 2008; 63:47-57.

30. Claudio L, Stingone JA, Godbold J. Prevalence of childhood asthma in urban communities: the impact of ethnicity and income. Ann Epidemiol 2006; 16:332-40

31. Eldeirawi KM, Persky VW. Associations of physician-diagnosed asthma with country of residence in the first year of life and other immigration-related factors: Chicago Asthma School Study. Ann Allergy Asthma Immunol 2007; 99:236-43.

32. Higgins PS, Wakefield D, Cloutier MM. Risk factors for asthma and asthma severity in nonurban children in Connecticut. Chest 2005; 128:3846-53.

33. Ledogar RJ, Penchaszadeh A, Garden CC, Iglesias G. Asthma and Latino cultures: different prevalence reported among groups sharing the same environment. Am J Public Health 2000; 90:929-35.

34. Lima RG, Pastorino AC, Casagrande RR, Sole D, Leone C, Jacob CM. Prevalence of asthma, rhinitis and eczema in 6-7 years old students from the western districts of São Paulo City, using the standardized questionnaire of the International Study of Asthma and Allergies in Childhood (ISAAC) phase IIIB. Clinics 2007; 62:225-34.

35. Shohat T, Green MS, Davidson Y, Livne I, Tamir R, Garty BZ. Differences in the prevalence of asthma and current wheeze between Jews and Arabs: results from a national survey of schoolchildren in Israel. Ann Allergy Asthma Immunol 2002; 89: 386-92.

37. Casagrande RR, Pastorino AC, Souza RG, Leone C, Sole D, Jacob CM. Prevalência de asma e fatores de risco em escolares da Cidade de São Paulo. Rev Saúde Pública 2008; 42:517-23.

38. Aligne CA, Auinger P, Byrd RS, Weitzman M. Risk factors for pediatric asthma. Contributions of poverty, race, and urban residence. Am J Respir Crit Care Med 2000; 162:873-7.

39. Berg J, Rachelefsky G, Jones CA, Tichacek MJ, Morphew $\mathrm{T}$. Identification of preschool children with asthma from low-income families in Los Angeles, CA. Ann Allergy Asthma Immunol 2004; 93:465-71.

40. Davis AM, Kreutzer R, Lipsett M, King G, Shaikh N. Asthma prevalence in Hispanic and Asian American ethnic subgroups: results from the California Healthy Kids Survey. Pediatrics 2006; 118:e363-70.

41. McDaniel M, Paxson C, Waldfogel J. Racial disparities in childhood asthma in the United States: evidence from the National Health Interview Survey, 1997 to 2003. Pediatrics 2006; 117:e868-77.

42. Smith LA, Hatcher-Ross JL, Wertheimer R, Kahn RS. Rethinking race/ethnicity, income, and childhood asthma: racial/ethnic disparities concentrated among the very poor. Public Health Rep 2005; 120:109-16. 
43. Chor D, Lima CRA. Aspectos epidemiológicos das desigualdades raciais em saúde no Brasil. Cad Saúde Pública 2005; 21:1586-94.

44. Jones CP. Levels of racism: a theoretic framework and a gardener's tale. Am J Public Health 2000; 90:1212-5.

45. Garcia E, Aristizabal G, Vasquez C, Rodriguez-Martinez CE, Sarmiento OL, Satizabal CL. Prevalence of and factors associated with current asthma symptoms in school children aged 6-7 and 13-14 yr old in Bogota, Colombia. Pediatr Allergy Immunol 2008; 19:307-14.

46. Demir AU, Karakaya G, Bozkurt B, Sekerel BE, Kalyoncu AF. Asthma and allergic diseases in schoolchildren: third cross-sectional survey in the same primary school in Ankara, Turkey. Pediatr Allergy Immunol 2004; 15:531-8.

47. Mellinger-Birdsong AK, Powell KE, Iatridis T, Bason J. Prevalence and impact of asthma in children, Georgia, 2000. Am J Prev Med 2003; 24:242-8.

48. Hertz-Picciotto I, Baker RJ, Yap PS, Dostal M, Joad JP, Lipsett M, et al. Early childhood lower respiratory illness and air pollution. Environ Health Perspect 2007; 115:1510-8.

49. Chatkin M, Menezes AM, Albernaz E, Victora CG, Barros FC. Fatores de risco para consultas em pronto-socorro por crianças asmáticas no Sul do Brasil. Rev Saúde Pública 2000; 34:491-8.

50. Lynch J, Kaplan G. Socioeconomic position. In: Berkman LF, Kawachi I, editors. Social epidemiology. New York: Oxford University Press; 2000; p. 13-35.
51. Galobardes B, Shaw M, Lawlor DA, Smith GD, Lynch J. Indicators of socioeconomic position. In: Oakes JM, Kaufman JS, editors. Methods in social epidemiology. San Francisco: Jossey-Bass; 2006; p. 47-85.

52. Prietsch SOM, Fischer GB, César JA, Lempek BS, Barbosa Jr. LV, Zogbi L, et al. Acute lower respiratory illness in under-five children in Rio Grande, Rio Grande do Sul State, Brazil: prevalence and risk factors. Cad Saúde Pública 2008; 24:1429-38.

53. Panico L, Bartley M, Marmot M, Nazroo JY, Sacker A, Kelly YJ. Ethnic variation in childhood asthma and wheezing illnesses: findings from the Millennium Cohort Study. Int J Epidemiol 2007; 36: 1093-102.

54. Cardoso MR, Cousens SN, de Goes-Siqueira LF, Alves FM, D'Angelo LA. Crowding: risk factor or protective factor for lower respiratory disease in young children? BMC Public Health 2004; 4:19.

55. Gonzales M, Malcoe LH, Myers OB, Espinoza J. Risk factors for asthma and cough among Hispanic children in the southwestern United States of America, 2003-2004. Rev Panam Salud Pública 2007; 21:274-81.

56. Victora CG, Vaughan JP, Barros FC, Silva AC, Tomasi E. Explaining trends in inequities: evidence from Brazilian child health studies. Lancet 2000; 356:1093-8.

57. Almeida MF, Barata RB, Montero CV, Silva ZP. Prevalência de doenças crônicas auto-referidas e utilização de serviços de saúde, PNAD/1998, Brasil. Ciênc Saúde Coletiva 2002; 7:743-56.

58. Viacava F, Dachs N, Travassos C. Os inquéritos domiciliares e o Sistema Nacional de Informações em Saúde. Ciênc Saúde Coletiva 2006; 11:863-9.

Recebido em 04/Jun/2009

Versão final reapresentada em 06/Abr/2010

Aprovado em 14/Jul/2010 